Revue d'histoire de l'Amérique française

REYUE D.HISTOIRE DE L'AMÉRIQUE FRANÇAISE

\title{
AXELROD, Paul, Making a Middle Class: Student Life in English \\ Canada During the Thirties. Montréal et Kingston, McGill-Queen's University Press, 1990. 269 p.
}

\section{Nicole Neatby}

Volume 45, numéro 2, automne 1991

URI : https://id.erudit.org/iderudit/304970ar

DOI : https://doi.org/10.7202/304970ar

Aller au sommaire du numéro

Éditeur(s)

Institut d'histoire de l'Amérique française

ISSN

0035-2357 (imprimé)

1492-1383 (numérique)

Découvrir la revue

Citer ce compte rendu

Neatby, N. (1991). Compte rendu de [AXELROD, Paul, Making a Middle Class: Student Life in English Canada During the Thirties. Montréal et Kingston, McGill-Queen's University Press, 1990. 269 p.] Revue d'histoire de l'Amérique française, 45(2), 259-261. https://doi.org/10.7202/304970ar d'utilisation que vous pouvez consulter en ligne.

https://apropos.erudit.org/fr/usagers/politique-dutilisation/ 
AXELROD, Paul, Making a Middle Class: Student Life in English Canada During the Thirties. Montréal et Kingston, McGill-Queen's University Press, 1990. 269 p.

Making a Middle Class suscite automatiquement la curiosité, voire même la reconnaissance, de ceux qui s'intéressent à l'histoire des étudiants universitaires canadiens-anglais. Comment pourrait-il en être autrement puisque cet ouvrage cherche à faire avancer un champ d'étude qui, encore aujourd'hui, demeure à l'étape des premiers défrichements?

Paul Axelrod s'aventure nettement en terre inexplorée lorsqu'il choisit d'analyser l'expérience des étudiants canadiens-anglais de neuf universités, représentant chaque région du pays durant la décennie de la Dépression. Mais l'aspect novateur de cette étude tient surtout au fait que l'auteur se propose d'aborder ce «narrowly defined (topic)» (p. 5) armé d'un large éventail de questions qui tiennent compte des intérêts des multiples branches de l'histoire sociale canadienne et des méthodes qu'elles ont explorées. Il veut en premier lieu produire une étude qui s'éloigne des approches traditionnelles, qui soit moins dépendante du discours des autorités universitaires et professorales et «more attuned to the sudent voice» (p. 17); cela lui permet de scruter «the collective life of a group considered relatively powerless» (p. 4). D'autre part, tout en ambitionnant de faire ressortir l'existence d'une «common student culture» (p. 4) au sein des universités canadiennes-anglaises, il projette aussi d'étudier «the unique culture of female students», en intégrant à son analyse les préoccupations de l'histoire des femmes. De façon générale, Paul Axelrod considère que son travail de recherche vise à apporter une contribution «to the social history of higher education, to the history of youth, to the history of the middle class, and to the history of the Depression» (p. 5).

Par de tels propos d'introduction, l'auteur ne peut faire autrement que de créer des attentes particulièrement élevées chez un public lecteur déjà avide de connaissances. Disons pour commencer qu'il réussit admirablement bien à nous présenter, sous de multiples facettes, le milieu étudiant canadienanglais des années trente. Il a fait un travail de synthèse remarquable, qui incorpore à bon escient les résultats de recherche tirés d'un vaste répertoire historiographique, dont les 80 pages de notes fournissent un aperçu. Tout au long de son analyse, Axelrod prend bien soin de faire ressortir les expériences distinctes des étudiantes, et cela donne lieu à une étude comparative des plus enrichissantes qui confirme incontestablement l'emprise de l'idéologie des «deux sphères» dans les milieux de haut savoir. Il se montre aussi très intéressé à la composition ethnique et religieuse du milieu étudiant canadienanglais, et fait valoir la présence dominante à l'université des jeunes blancs 
anglo-saxons et chrétiens. L'attention soutenue portée au sort que l'on réserve à l'époque aux étudiants juifs permet à l'auteur de nous rappeler de façon éloquente combien les milieux universitaires canadiens sont loin d'avoir été à l'abri de l'intolérance et du racisme. Notons que Paul Axelrod sait aussi interroger ses sources de manière à nous faire sentir l'impact du contexte régional sur l'évolution des différentes universités du pays. Par ailleurs, tous ceux qui doutent encore du «caractère distinct» du Canada anglais seront bel et bien obligés de se détromper à la lumière des multiples comparaisons que l'auteur établit avec les développements qui ont cours aux États-Unis à la même époque.

Si son regard «polyvalent» fournit un aperçu tout en nuances du milieu étudiant canadien-anglais, il lui permet aussi d'émettre des généralisations des plus éclairantes autour desquelles s'articule l'ensemble de ses recherches. C'est ainsi que P. Axelrod réussit à nous convaincre que les étudiants qui décident de s'inscrire à l'université durant les années difficiles de la Dépression sont majoritairement issus des classes moyennes. Il prend bien soin d'expliquer que dans le contexte de la société canadienne, une telle identification ne signifie en rien l'appartenance à un milieu fortuné. L'analyse fouillée qu'il a le mérite de fournir en appendice sur ce qu'il entend par «classes moyennes», jumelée à l'information qu'il a su glaner dans les sources fournissant la profession des pères d'étudiants, nous amène à rejeter «the caricatures of rich, carefree students» (p. 28) et à suivre l'auteur lorsqu'il avance que tout en constituant «a select group» (p. 37), «most students came from modest middle-class as opposed to exceptionally privileged upper-class families» (p. 24).

Mais P. Axelrod va plus loin et affirme que dans l'ensemble les étudiants des années trente se servent de leur passage à l'université pour obtenir, maintenir ou améliorer leur place au sein de cette classe moyenne (p. 35). En faisant ressortir les nouveaux courants qui transforment l'économie du pays au début du siècle, l'auteur démontre effectivement que les universités sont de plus en plus appelées à contribuer à la formation d'une main-d'œuvre spécialisée susceptible d'attirer les membres de la classe moyenne.

L'analyse est peut-être moins convaincante quand l'auteur soutient que ces institutions transmettent aussi à leurs populations étudiantes les valeurs et les priorités propres aux classes moyennes, à savoir le respect de l'autorité, le sens du devoir, les bienfaits de la sécurité financière. Il est vrai que son examen minutieux du discours tenu par les autorités universitaires de l'époque laisse certainement croire à une telle vocation de socialisation. Toutefois lorsque l'auteur tente de démontrer que les étudiants ont eux-mêmes participé «actively in their own socialization to the middle-class values embraced by parents and teachers» (p. 19), il produit une analyse qui ne laisse pratiquement pas de place à la voix étudiante qu'il se proposait pourtant de nous faire entendre; il ne donne d'ailleurs que peu d'importance aux points de vue des étudiants dissidents et foncièrement autonomes. Il nous présente les universités des années trente essentiellement comme des agences de contrôle social (p. 171) qui réussissent bel et bien à produire des citoyens eux-mêmes disposés à s'intégrer au milieu social de la classe moyenne 
auquel ils sont destinés. Pourtant cette même étude contient un chapitre fascinant, qui révèle contre toute attente qu'une composante de la population étudiante $(5 \%)$ (p. 135) s'active à effectuer des réformes sociales et politiques, certains allant même jusqu'à s'intéresser à l'idéologie non-conformiste par excellence, le communisme. Le lecteur demeure intrigué par cette apparente «schizophrénie» du corps étudiant de l'époque. D'où sortent ces jeunes activistes? Partageaient-ils les mêmes origines sociales que leurs collègues plus conformistes? Ont-ils eux aussi fini par rejoindre les rangs de la classe moyenne? Peut-être était-il difficile pour l'auteur de rendre compte de leurs idéaux et de leur comportement distincts, mais ces questions sans réponse méritaient néanmoins d'être davantage explorées.

Il ne fait aucun doute que Making a Middle Class est un ouvrage qui provoque la réflexion. Son grand mérite est d'avoir effectivement mis en pratique les ambitions de l'histoire sociale: faire converger une multiplicité de développements sociaux d'une grande portée pour comprendre l'évolution d'un groupe ou de phénomènes relativement circonscrits. Il devient ainsi un modèle incontournable pour ceux qui exploreront le monde étudiant d'autres décennies. 